\title{
Lipid emulsion attenuates the vasodilation induced by a toxic dose of a calcium channel blocker through its partitioning into the lipid phase
}

\author{
Seong-Ho Ok ${ }^{1,7,}$, Soo Hee Lee ${ }^{2,7,}$, Hyun-Jin Kim ${ }^{3,4}$, Jeong-Min Hong ${ }^{5}$, Ji-Yoon Kim ${ }^{6}$, Sung Il \\ $\mathrm{Bae}^{6}$, Yunsik Shin ${ }^{6}$ and Ju-Tae Sohn ${ }^{2,7}$ \\ ${ }^{1}$ Department of Anesthesiology and Pain Medicine, Gyeongsang National University Changwon Hospital, Changwon, \\ Republic of Korea \\ ${ }^{2}$ Department of Anesthesiology and Pain Medicine, Gyeongsang National University School of Medicine, Gyeongsang \\ National University Hospital, Gyeongsangnam-do, Republic of Korea \\ ${ }^{3}$ Division of Applied Life Sciences (BK21 plus), Gyeongsang National University, Gyeongsang, Republic of Korea \\ ${ }^{4}$ Department of Food Science \& Technology, Institute of Agriculture and Life Science, Gyeongsang National University, \\ Gyeongsang, Republic of Korea \\ ${ }^{5}$ Department of Anesthesia and Pain Medicine, Pusan National University Hospital, Biomed Research Institute, Pusan \\ National University School of Medicine, Busan, Republic of Korea \\ ${ }^{6}$ Department of Anesthesiology and Pain Medicine, Gyeongsang National University Hospital, Gyeongsangnam-do, Republic \\ of Korea \\ ${ }^{7}$ Institute of Health Sciences, Gyeongsang National University, Jinju-si, Republic of Korea
}

\begin{abstract}
The present in vitro study examined whether lipid emulsion attenuates the vasodilation evoked by toxic doses of calcium channel blockers (bepridil, verapamil, nifedipine and diltiazem) via their partitioning into the lipid phase. The effects of the calcium channel blockers alone, the lipid emulsion and calcium channel blocker mixture, and the centrifuged aqueous extract (CAE) obtained from ultracentrifugation of the lipid emulsion and calcium channel blocker mixture on isolated endothelium-denuded rat aortas precontracted with phenylephrine were observed. The effects of lipid emulsion on calcium channel blocker concentration in the Krebs solution were examined using ultraperformance liquid chromatography. A mixture of lipid emulsion with either bepridil or verapamil and the corresponding CAE more effectively attenuated vasodilation than either bepridil or verapamil alone, whereas the vasodilation induced by the mixture of lipid emulsion and either bepridil or verapamil was not significantly different from that induced by the corresponding CAE. The magnitude of the lipid emulsion-mediated reduction in vasodilation and calcium channel blocker concentration was as follows: bepridil > verapamil > nifedipine or diltiazem. These results suggest that lipid emulsion attenuates vasodilation induced by a toxic dose of bepridil and verapamil, seemingly through partitioning of the calcium channel blocker into the lipid phase.
\end{abstract}

Key words: Verapamil - Lipid emulsion - Vasodilation - Partitioning - Centrifuged aqueous extract

Abbreviations: HPLC, high-performance liquid chromatography; L-NAME, $\mathrm{N}^{\mathrm{W}}$-nitro-L-arginine methyl ester; UPLC-Q-TOF MS, ultraperformance liquid chromatography-quadrupole time-of-flight mass spectrometry; CAE, centrifuged aqueous extract.

\footnotetext{
* These authors contributed equally to the work.

Correspondence to: Ju-Tae Sohn, Department of Anesthesiology and Pain Medicine, Gyeongsang National University School of Medicine, Gyeongsang National University Hospital, 79 Gangnam-ro, Jinju-si, 52727, Republic of Korea

E-mail: jtsohn@gnu.ac.kr
} 


\section{Introduction}

Lipid emulsion is reportedly effective in treating cardiovascular collapse induced by toxic doses of either local anaesthetic or other highly lipid-soluble drugs (Log [octanol/ water partition coefficient] > 2.0), including verapamil and tricyclic antidepressants (Weinberg 2012; Cao et al. 2015; Ok and Sohn 2018). Lipid emulsion is more effective at attenuating verapamil-induced toxicity than attenuating toxicity induced by other calcium channel blockers (St-Onge et al. 2014; Cao et al. 2015). In addition, according to case reports and an analysis of reported cases undergoing lipid emulsion treatment, intravenous and intraosseous administration of lipid emulsion effectively alleviates the cardiovascular depression induced by toxic doses of verapamil in adults and children, which seems to be associated with a lower verapamil concentration following administration of lipid emulsion (French et al. 2011a; Liang et al. 2011; Presley and Chyka 2013; Sampson and Bedy 2015). Among the proposed underlying mechanisms associated with lipid emulsion treatment as a non-specific antidote, the scavenging effect (lipid sink and shuttle) is most widely accepted and states that a highly lipid-soluble drug is absorbed into the lipid emulsion of plasma from tissue affected by the toxic dose of the drug (partitioning) and redistributed into muscle and liver for detoxification (Weinberg 2012; Ok et al. 2018a). Lipid emulsion attenuates the inhibition of cardiomyocyte contractility induced by verapamil and reduces the verapamil concentration (Kryshtal et al. 2016). Furthermore, the treatment with a lipid emulsion alleviates vasodilation evoked by calcium channel blockers, which appears to be positively correlated with the lipid solubility (oil/Ringer partition coefficient) of calcium channel blockers and the order of uptake of calcium channel blockers into rat aortic vascular smooth muscle cells (Pang and Sperelakis 1984; Ok et al. 2018b). The centrifuged aqueous extract (CAE) lacking the upper lipid phase (layer) obtained after the ultracentrifugation of a mixture of the highly lipid-soluble local anaesthetic bupivacaine and lipid emulsion produces a smaller reduction in mean arterial pressure than bupivacaine alone, but a significant difference in the mean arterial pressure was not observed between subjects administered the CAE and subjects given the bupivacaine and lipid emulsion mixture (Hori et al. 2015). Moreover, the CAE without the upper lipid phase obtained by ultracentrifugation was shown to partially recover the attenuated fast sodium current induced by bupivacaine alone in rat cardiomyocytes (Wagner et al. 2014). These previous reports suggest that lipid emulsion-mediated recovery is associated with greater partitioning of the highly lipid-soluble bupivacaine into lipid emulsions (Wagner et al. 2014; Hori et al. 2015). However, the detailed mechanism contributing to the inhibitory effect of lipid emulsion on the vasodilation evoked by toxic doses of calcium channel block- ers remains unknown. Based on previous reports, we tested the hypothesis that lipid emulsion inhibits the vasodilation induced by a toxic dose of calcium channel blockers through the partitioning of calcium channel blockers into the lipid phase of lipid emulsions (Pang and Sperelakis 1984; Wagner et al. 2014; Hori et al. 2015; Ok et al. 2018a, 2018b). First, the objective of this study was to examine whether a lipid emulsion (Intralipid ${ }^{\circ}$ ) inhibits vasodilation induced by toxic doses of calcium channel blockers through the partitioning of the calcium channel blocker into the lipid phase of lipid emulsions using ultracentrifugation to separate a mixture of lipid emulsion and calcium channel blockers into an upper lipid phase and lower CAE. Second, we investigated the effect of lipid emulsion on the concentration of calcium channel blockers.

\section{Materials and Methods}

All experimental protocols were approved by the Institutional Animal Care and Use Committee of Gyeongsang National University. All experimental procedures were performed according to the Guide for the Care and Use of Laboratory Animals approved by the Institute for Laboratory Animal Research.

\section{Preparation of isolated rat aorta and isometric tension measurement}

Isolated rat thoracic aorta was prepared for tension measurement as described previously (Ok et al. 2018b). Male Sprague-Dawley rats (body weight: 250 to $300 \mathrm{~g} ; n=35$ ) were anaesthetized with $100 \%$ carbon dioxide. The surrounding connective tissue and fat were removed from the descending thoracic aorta bathed in Krebs solution under a microscope. The Krebs solution was composed of sodium chloride (118 mmol/l), sodium bicarbonate $(25 \mathrm{mmol} / \mathrm{l})$, glucose $(11 \mathrm{mmol} / \mathrm{l})$, potassium chloride $(4.7 \mathrm{mmol} / \mathrm{l})$ and monopotassium phosphate $(1.2 \mathrm{mmol} / \mathrm{l})$. The isolated rat aorta was cut into segments 3 to $4 \mathrm{~mm}$ in length. The endothelium of the isolated rat aorta was removed by inserting a 25-gauge needle into the isolated aortic lumen and rolling the aorta forward and backward for approximately 10 seconds. The isolated aortic rings were suspended in a Grass isometric transducer (FT-03, Grass Instrument, Quincy, MA, USA). The 3.0-g resting tension was maintained for $120 \mathrm{~min}$, and the Krebs solution was replaced with fresh Krebs solution every $30 \mathrm{~min}$. Phenylephrine $\left(10^{-8} \mathrm{~mol} / \mathrm{l}\right)$ was added to the organ bath to induce contraction of isolated rat aorta without the endothelium. After $10^{-8} \mathrm{~mol} / \mathrm{l}$ phenylephrine induced sustained and stable vasoconstriction, acetylcholine $\left(10^{-5} \mathrm{~mol} / \mathrm{l}\right)$ was added to the organ bath to confirm endothelial denudation. A less than $15 \%$ acetylcholine-induced 
relaxation from contraction evoked by phenylephrine was regarded as indicative of an endothelium-denuded aorta in this experiment. The isolated rat aortas showing acetylcholineinduced relaxation from phenylephrine-induced contraction were then washed with fresh Krebs solution every $10 \mathrm{~min}$ for $30 \mathrm{~min}$, and the baseline resting tension was recovered. The little remaining endothelium after endothelial denudation may affect endothelial nitric oxide production. Furthermore, nifedipine and diltiazem enhance nitric oxide production in endothelial cells, whereas verapamil has no effect (Ding and Vaziri 2000). In addition, lipid emulsion inhibits acetylcholine-induced nitric oxide-mediated relaxation (Ok et al. 2015). Thus, as these may be confounding factors in the interpretation of the results obtained from the current study, we pretreated endothelium-denuded aortic rings with the nitric oxide synthase inhibitor $\mathrm{N}^{\mathrm{W}}$-nitro-L-arginine methyl ester (L-NAME, $10^{-4} \mathrm{~mol} / \mathrm{l}$ ) to avoid these confounding factors, with a particular focus on partitioning calcium channel blockers (Ding and Vaziri 2000; Ok et al. 2015).

\section{Experimental protocols}

After phenylephrine $\left(10^{-7} \mathrm{~mol} / \mathrm{l}\right)$ produced a sustained and stable contraction in isolated endothelium-denuded rat aorta pretreated with L-NAME $\left(10^{-4} \mathrm{~mol} / \mathrm{l}\right)$, calcium channel blockers $\left(10^{-5} \mathrm{~mol} / \mathrm{l}\right.$ bepridil, $10^{-7} \mathrm{~mol} / \mathrm{l}$ verapamil, or $3 \times 10^{-7} \mathrm{~mol} / \mathrm{l}$ nifedipine and diltiazem) alone, a mixture of the lipid emulsion $(0.998 \%)$ and calcium channel blockers $\left(10^{-5} \mathrm{~mol} / \mathrm{l}\right.$ bepridil, $10^{-7} \mathrm{~mol} / \mathrm{l}$ verapamil, or $3 \times$ $10^{-7} \mathrm{~mol} / \mathrm{l}$ nifedipine and diltiazem), and a corresponding CAE obtained from a mixture of the lipid emulsion and calcium channel blockers was added to the organ bath to examine the effects on the isolated endothelium-denuded rat aorta precontracted with $10^{-7} \mathrm{~mol} / \mathrm{l}$ phenylephrine. Then, the vascular tone change induced by these treatments was monitored for $1 \mathrm{~h}$. The maximal vasodilation in each treatment condition was selected and was compared across treatment with calcium channel blocker alone, a mixture of lipid emulsion and calcium channel blocker, and CAE. The isolated rat aortas, which were obtained from the same rat, were treated with three formulations (calcium channel blocker alone, a mixture of the calcium channel blocker and the lipid emulsion, and corresponding CAE) of each calcium channel blocker (bepridil, verapamil, nifedipine or diltiazem). Seven independent experiments were performed using the three formulations of each calcium channel blocker. Detailed descriptions of the experimental protocols are provided below. After phenylephrine $\left(10^{-7} \mathrm{~mol} / \mathrm{l}\right)$ produced a sustained and stable contraction in endothelium-denuded rat aortas pretreated with $10^{-4} \mathrm{~mol} / \mathrm{l}$ L-NAME in $9.5 \mathrm{ml}$ of Krebs solution, $500 \mu \mathrm{l}$ of Krebs solution containing each calcium channel blocker alone $\left(2 \times 10^{-4} \mathrm{~mol} / \mathrm{l}\right.$ bepridil, $2 \times 10^{-6} \mathrm{~mol} / \mathrm{l}$ verapamil, or $6 \times$

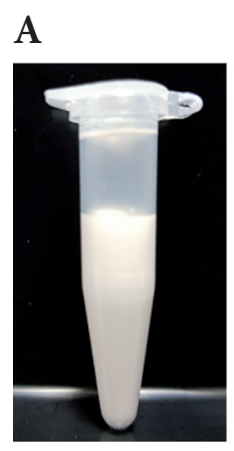

B

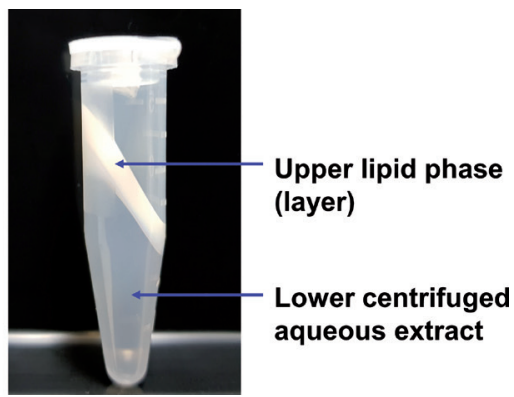

Figure 1. The differences in lipid emulsion containing verapamil $\left(2 \times 10^{-6} \mathrm{~mol} / \mathrm{l}\right)$ before (A) and after (B) 20-min ultracentrifugation $\left(75,000 \times g, 4^{\circ} \mathrm{C}\right)$.

$10^{-6} \mathrm{~mol} / \mathrm{l}$ nifedipine and diltiazem), $500 \mu \mathrm{l}$ of lipid emulsion (19.96\%) (Fig. 1A) containing each calcium channel blocker $\left(2 \times 10^{-4} \mathrm{~mol} / \mathrm{l}\right.$ bepridil, $2 \times 10^{-6} \mathrm{~mol} / \mathrm{l}$ verapamil, or $6 \times 10^{-6} \mathrm{~mol} / \mathrm{l}$ nifedipine and diltiazem), and $500 \mu \mathrm{l}$ of CAE (Fig. 1B) obtained from a mixture of lipid (19.96\%) and each calcium channel blocker $\left(2 \times 10^{-4} \mathrm{~mol} / \mathrm{l}\right.$ bepridil, $2 \times 10^{-6} \mathrm{~mol} / \mathrm{l}$ verapamil, or $6 \times 10^{-6} \mathrm{~mol} / \mathrm{l}$ nifedipine and diltiazem) using ultracentrifugation was added to the organ bath to produce the calcium channel blocker alone, mixture of calcium channel blocker and lipid emulsion, and corresponding CAE, respectively. The toxic concentration of calcium channel blockers used in this experiment was chosen based on a previous report (Schulz and Schmoldt. 2003). A mixture (Fig. 1A) of lipid emulsion and calcium channel blocker was prepared by mixing $10 \mathrm{cc}$ of lipid emulsion $(19.96 \%)$ containing each calcium channel blocker $\left(2 \times 10^{-4} \mathrm{~mol} / \mathrm{l}\right.$ bepridil, $2 \times 10^{-6} \mathrm{~mol} / \mathrm{l}$ verapamil, or $6 \times$ $10^{-6} \mathrm{~mol} / \mathrm{l}$ nifedipine and diltiazem $)$ for $30 \mathrm{~min}\left(37^{\circ} \mathrm{C}\right.$; $60 \mathrm{rpm}$ ) using a Mylab Multi Mixer (\#SLRM-3) (Seoulin Bioscience, Bundang, Korea). CAE (Fig. 1B) was obtained by ultracentrifuging $\left(75,000 \times g, 4^{\circ} \mathrm{C}\right)$ a mixture of lipid emulsion $(19.96 \%)$ and each calcium channel blocker $\left(2 \times 10^{-4} \mathrm{~mol} / \mathrm{l}\right.$ bepridil, $2 \times 10^{-6} \mathrm{~mol} / \mathrm{l}$ verapamil, or $6 \times$ $10^{-6} \mathrm{~mol} / \mathrm{l}$ nifedipine and diltiazem) for $20 \mathrm{~min}$ using an Optimal TM MAX-XP ultracentrifuge (Beckman Coulter Life Sciences, Indianapolis, IN, USA) and removing the upper lipid phase (layer) from the resulting solution. The recommended plasma lipid emulsion concentration for the partitioning effect in cases of oral overdose-induced intoxication is $1 \%$ triglyceride $(1000 \mathrm{mg} / \mathrm{dl}$ ) (Fettiplace et al. 2015). In addition, a previous study suggested that $1 \%$ lipid emulsion attenuates vasodilation induced by a toxic dose of a calcium channel blocker (Ok et al. 2018b). Therefore, a $0.998 \%$ lipid emulsion concentration, which corresponds to an approximately $1 \%$ plasma lipid emulsion concentration, was chosen in this experiment using $20 \%$ Intralipid $^{\circledR}$ as the lipid emulsion (Fettiplace et al. 2015; Ok et al. 2018b). 
The effect of lipid emulsion (1\%) on phenylephrineinduced contraction in isolated endothelium-denuded rat aorta was investigated. After phenylephrine $\left(10^{-7} \mathrm{~mol} / \mathrm{l}\right)$ produced a sustained and stable contraction, lipid emulsion (1\%) was added to the organ bath, and vascular tone was monitored for $1 \mathrm{~h}$.

\section{Effect of lipid emulsion on the concentration of bepridil, verapamil and diltiazem in the Krebs solution}

Ultraperformance liquid chromatography was performed to measure the concentration of calcium channel blockers as described in a previous experiment (Lee et al. 2018). Calcium channel blockers $\left(10^{-5} \mathrm{~mol} / \mathrm{l}\right.$ bepridil, $10^{-7} \mathrm{~mol} / \mathrm{l}$ verapamil and $3 \times 10^{-7} \mathrm{~mol} / \mathrm{l}$ diltiazem) dissolved in the Krebs solution were mixed with Intralipid ${ }^{\circ}(1 \%)$ on a rotator for $30 \mathrm{~min}$ to emulsify the calcium channel blockers and lipids. After centrifugation at $75,000 \times g$ for $40 \mathrm{~min}$, the unemulsified calcium channel blocker in the aqueous layer was measured by ultraperformance liquid chromatography-quadrupole time-of-flight mass spectrometry (UPLC-Q-TOF MS; Waters, Milford, MA, USA). The unemulsified calcium channel blocker samples were injected into an Acquity UPLC BEH $\mathrm{C}_{18}$ column $(100 \times 2.1 \mathrm{~mm}, 1.7 \mu \mathrm{m}$; Waters $)$ equilibrated with water containing $0.1 \%$ formic acid and eluted with a linear acetonitrile containing a $0.1 \%$ formic acid gradient $(1-100 \%)$ at a flow rate of $0.35 \mathrm{ml} / \mathrm{min}$ for $5 \mathrm{~min}$. The eluted calcium channel blockers were analyzed by Q-TOF MS (Waters) using the multiple reaction monitoring (MRM) and positive electrospray ionization modes. The capillary and sampling cone voltages were set at $3 \mathrm{kV}$ and $30 \mathrm{~V}$, respectively. The desolvation temperature and flow rate were $100^{\circ} \mathrm{C}$ and $800 \mathrm{l} / \mathrm{h}$, respectively, and the source temperature was set at $400^{\circ} \mathrm{C}$. Lock spray with leucine-enkephalin $(556.2771 \mathrm{Da})$ was used at a frequency of $10 \mathrm{~s}$ to ensure reproducibility and accuracy for all analyses. For quantitative analysis of calcium channel blockers, MRM was used, and the precursor and product ions for calcium channel blockers were 289.21 and 140.13, respectively. All mass data were collected and analyzed by UIFI version 1.8.2 (Waters).

\section{Effect of lipid emulsion on the nifedipine concentration}

High-performance liquid chromatography (HPLC) was used to measure the nifedipine concentration because nifedipine is thermally unstable and photosensitive (Kleinbloesem et al. 1984; Suzuki et al. 1985). The high temperature employed in UPLC-Q-TOF MS and the photosensitivity of nifedipine may result in the degradation of nifedipine into its pyridine metabolite (Kleinbloesem et al. 1984; Suzuki et al. 1985). In addition, HPLC accurately detects the concentration of nifedipine to more than $10^{-5} \mathrm{~mol} / \mathrm{l}$, which is higher than the $3 \times 10^{-7} \mathrm{~mol} / \mathrm{l}$ nifedipine used in the tension study.
Thus, $20 \mu \mathrm{g} / \mathrm{ml}\left(5.7 \times 10^{-5} \mathrm{~mol} / \mathrm{l}\right)$ nifedipine, which is approximately 190 times higher than the concentration used in tension study $\left(3 \times 10^{-7} \mathrm{~mol} / \mathrm{l}\right)$, was chosen to examine the effect of lipid emulsion on the nifedipine concentration in the current study. We prepared sample solutions by centrifuging the lipid emulsion (1\%) containing $20 \mu \mathrm{g} / \mathrm{mL}$ (approximately $\left.5.7 \times 10^{-5} \mathrm{~mol} / \mathrm{l}\right)$ nifedipine at $75,000 \times g$ for $20 \mathrm{~min}$ at $4^{\circ} \mathrm{C}$ and removing the upper lipid phase to obtain the CAE. The stock solution of nifedipine was prepared at $2.28 \mathrm{mg} / \mathrm{ml}$ in dimethyl sulfoxide (DMSO). Standard solutions for the calibration curve were prepared by diluting appropriate portions of stock solution with distilled water. Calibration curves were obtained with four standard solutions containing 1, 10, 20 and $50 \mu \mathrm{g} / \mathrm{ml}$ nifedipine in distilled water, and linear regression analysis was performed. HPLC analysis was performed using an ACE C18-AR column ( $250 \mathrm{~mm} \times 4.6 \mathrm{~mm}$ I.D, $5 \mu \mathrm{m}$ particle size, $10 \mathrm{~nm}$ pore size) (Advanced Chromatography Technologies Ltd. Aberdeen, Scotland) and 1260 Infinity II LC Systems (Agilent Technologies, INC. Santa Clara, CA, USA) maintained at $30^{\circ} \mathrm{C}$ as described previously (Chung et al. 2011). The mobile phases were (A) water and (B) acetonitrile, with the following gradient: $5 \% \mathrm{~B}(0-2 \mathrm{~min}), 5-60 \%$ B (2-15 min), 60\% B (15-18 min), and 60-5\% B (18-20 min) (all $\mathrm{v} / \mathrm{v}$ ) at $1 \mathrm{ml} / \mathrm{min}$. Eluates were monitored $v i a$ a photodiode array detector at $210,254,280$, and $320 \mathrm{~nm}$, and the scans at $210 \mathrm{~nm}$ were recorded. Authentic nifedipine was used as a standard for identification and quantification.

\section{Drugs}

All chemical agents were commercially available and of the highest purity. L-NAME, acetylcholine, nifedipine, verapamil, diltiazem, bepridil and formic acid were obtained from Sigma Aldrich (St. Louis, Missouri, USA), and the $20 \%$ Intralipid used in this experiment was obtained from Fresenius Kabi Korea (Seoul, Korea). Acetonitrile and water for HPLC were obtained from Honeywell Burdick \& Jackson (Ulsan, Korea). Nifedipine and bepridil were dissolved in DMSO. The other drugs were dissolved in distilled water.

\section{Statistical analysis}

The data are expressed as the mean \pm SD. The vasodilation induced by calcium channel blocker alone, a mixture of lipid emulsion and calcium channel blocker, and CAE on the phenylephrine-induced contraction of isolated endothelium-denuded rat aorta was expressed as the percentage of inhibition of maximal contraction induced by phenylephrine. A normality test regarding the data from the isometric tension study was performed by Shapiro-Wilk tests using Prism 5.0 (GraphPad, Software, San Diego, CA, USA). The effect of calcium channel blocker alone, a mixture of lipid emulsion and calcium channel blocker and CAE on 
the phenylephrine-induced contraction was analysed using one-way analysis of variance (ANOVA) followed by Bonferroni's multiple comparison test. The effect of lipid emulsion on phenylephrine-induced contraction was analysed using a linear mixed effect model. The effect of lipid emulsion on the concentration of bepridil, verapamil, nifedipine and diltiazem was analysed using one-way ANOVA followed by Bonferroni's multiple comparison test or unpaired Student's $t$-test. The sample size for the isometric tension study was calculated using resource equation methods (Charan and Kantharia. 2013). The value $p<0.05$ was considered statistically significant.

\section{Results}

Bepridil $\left(10^{-5} \mathrm{~mol} / \mathrm{l}\right)$ produced vasodilation in isolated endothelium-denuded rat aorta ( $48 \pm 6 \%$; Fig. $2 \mathrm{~A}$ ). The mixture of lipid emulsion $(0.998 \%)$ and bepridil $\left(10^{-5} \mathrm{~mol} / \mathrm{l}\right)$ or the CAE obtained from ultracentrifugation of the mixture produced remarkably less vasodilation than bepridil $\left(10^{-5} \mathrm{~mol} / \mathrm{l}\right)$ alone ( $p<0.001$; Fig. $2 \mathrm{~A}$ ). However, vasodilation induced by the mixture of the lipid emulsion $(0.998 \%)$ and bepridil $\left(10^{-5} \mathrm{~mol} / \mathrm{l}\right)$ was not significantly different from that induced by the corresponding CAE ( $p=0.42$; Fig. $2 \mathrm{~A})$. Verapamil $\left(10^{-7} \mathrm{~mol} / \mathrm{l}\right)$ produced vasodilation in isolated endotheliumdenuded rat aorta (29 $\pm 5 \%$; Fig. $2 \mathrm{~B})$. The mixture of lipid emulsion $(0.998 \%)$ and verapamil $\left(10^{-7} \mathrm{~mol} / \mathrm{l}\right)$ or the corresponding $\mathrm{CAE}$ produced moderately less vasodilation than verapamil $\left(10^{-7} \mathrm{~mol} / \mathrm{l}\right)$ alone $(p<0.001$; Fig. $2 \mathrm{~B})$. However, vasodilation induced by the mixture of lipid emulsion $(0.998 \%)$ and verapamil $\left(10^{-7} \mathrm{~mol} / \mathrm{l}\right)$ was not significantly different from vasodilation induced by the corresponding CAE ( $p=0.82$; Fig. 2 B). Nifedipine $\left(3 \times 10^{-7} \mathrm{~mol} / \mathrm{l}\right)$ produced vasodilation in isolated endothelium-denuded rat aorta (51 \pm $3 \%$; Fig. $2 \mathrm{C}$ ). The mixture ( $p=0.03$; Fig. $2 \mathrm{C}$ ) of lipid emulsion $(0.998 \%)$ and nifedipine $\left(3 \times 10^{-7} \mathrm{~mol} / \mathrm{l}\right)$ or the corresponding CAE ( $p<0.001$; Fig. $2 \mathrm{C}$ ) produced slightly less vasodilation than nifedipine $\left(3 \times 10^{-7} \mathrm{~mol} / \mathrm{l}\right)$ alone. In addition, the CAE produced slightly less vasodilation than the mixture of lipid emulsion $(0.998 \%)$ and nifedipine $\left(3 \times 10^{-7} \mathrm{~mol} / \mathrm{l}\right)$ $\left(p<0.001\right.$; Fig. 2C). Diltiazem $\left(3 \times 10^{-7} \mathrm{~mol} / \mathrm{l}\right)$ produced vasodilation in isolated endothelium-denuded rat aorta (34 $\pm 8 \%$; Fig. 2D). However, vasodilation induced by the mixture $(p=0.97)$ of lipid emulsion $(0.998 \%)$ and diltiazem $\left(3 \times 10^{-7} \mathrm{~mol} / \mathrm{l}\right)$ or that induced by the corresponding CAE $(p=0.07)$ was not significantly different from the vasodilation induced by diltiazem alone $\left(3 \times 10^{-7} \mathrm{~mol} / \mathrm{l}\right.$; Fig. 2D). The calcium channel blockers listed in decreasing order of the inhibitory effect of lipid emulsion $(0.998 \%)$ on the maximal vasodilation evoked by calcium channel blockers alone were as follows: bepridil $(81 \pm 3 \%)>$ verapamil $(37 \pm 10 \%)$ $>$ nifedipine $(10 \pm 4 \%)$ or diltiazem $(10 \pm 13 \%)(p<0.001$;
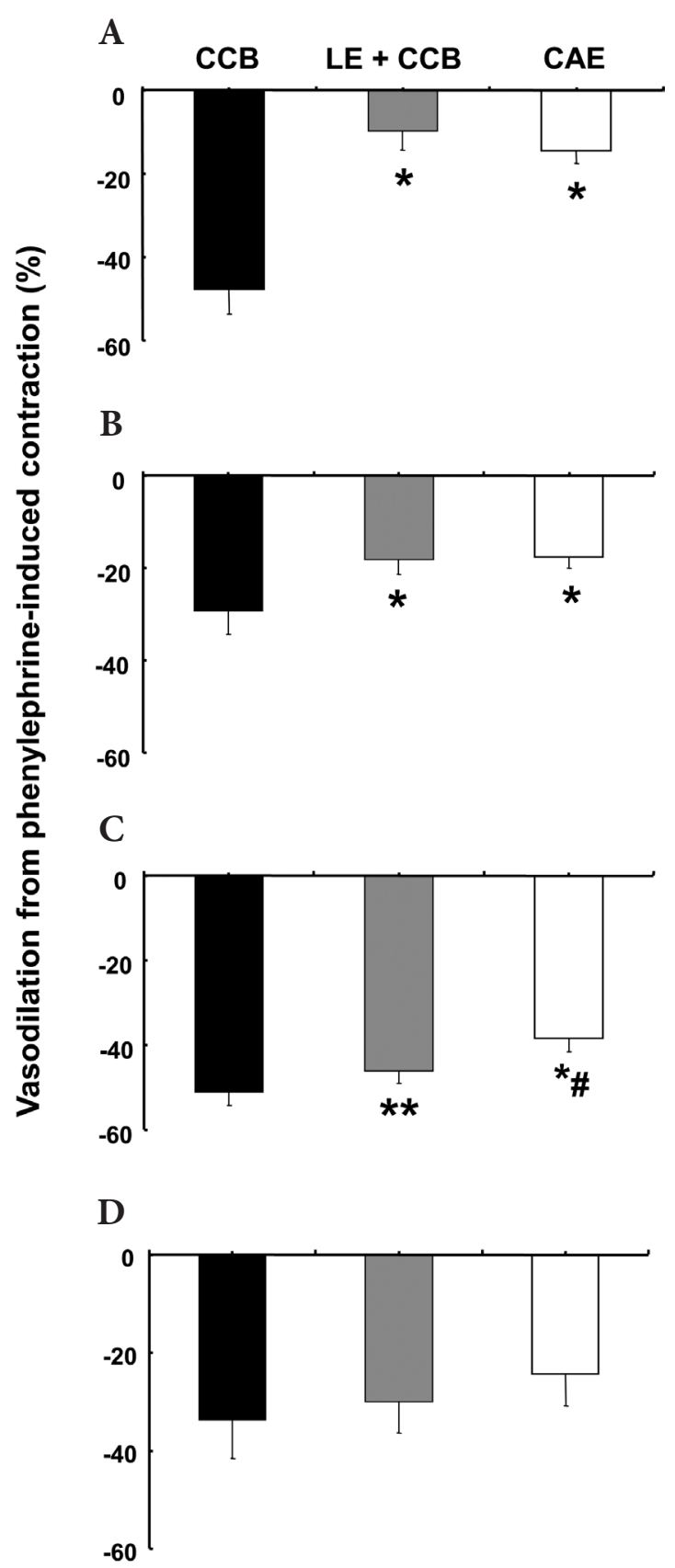

Figure 2. Effects of calcium channel blockers (CCB, $10^{-5} \mathrm{~mol} / \mathrm{l}$ bepridil (A), $10^{-7} \mathrm{~mol} / \mathrm{l}$ verapamil (B), $3 \times 10^{-7} \mathrm{~mol} / \mathrm{l}$ nifedipine (C) and $3 \times 10^{-7} \mathrm{~mol} / \mathrm{l}$ diltiazem (D); $n=7$ ) alone, a mixture $(n=7)$ of $0.998 \%$ lipid emulsion (LE) and CCB, and the corresponding centrifuged aqueous extract (CAE, $n=7$ ) obtained from ultracentrifugation of the mixture of LE and $\mathrm{CCB}$ on the endothelium-denuded rat aortas precontracted with $10^{-7} \mathrm{~mol} / \mathrm{l}$ phenylephrine. The data are shown as the mean \pm $\mathrm{SD}$ and expressed as the percentage of vasodilation (inhibition) of phenylephrine-induced contraction; $n$ indicates the number of biological replicates (independent experiments). ${ }^{*} p<0.001$, ${ }^{* *} p<0.05$ versus CCB alone; $\# p<0.001$ versus $\mathrm{LE}(0.998 \%)+$ nifedipine $\left(3 \times 10^{-7} \mathrm{~mol} / \mathrm{l}\right)$. 
Fig. 3A). However, the lipid emulsion (1\%)-induced reduction in nifedipine-induced vasodilation was not significantly different from that in diltiazem-induced vasodilation (Fig. $3 \mathrm{~A})$. Lipid emulsion (1\%) itself had no effect on the contraction induced by phenylephrine $\left(10^{-7} \mathrm{~mol} / \mathrm{l}\right)$ in isolated endothelium-denuded rat aorta (Fig. 3B).

Lipid emulsion (1\%) significantly decreased the concentration of bepridil $\left(10^{-5} \mathrm{~mol} / \mathrm{l}\right)$, verapamil $\left(10^{-7} \mathrm{~mol} / \mathrm{l}\right)$, nifedipine $(20 \mu \mathrm{g} / \mathrm{ml})$ and diltiazem $\left(3 \times 10^{-7} \mathrm{~mol} / \mathrm{l}\right)(p<$ 0.001 versus calcium channel blocker alone; Fig. 4$)$. In addition, the magnitude of the lipid emulsion (1\%)-mediated reduction in calcium channel blocker concentration was as follows (Fig. 4): bepridil $(80 \pm 3 \%)>$ verapamil $(27 \pm 2 \%)$ $>$ diltiazem $(8 \pm 5 \%)$ or nifedipine $(3.5 \pm 0.3 \%)$. In addition, the lipid emulsion (1\%)-mediated reduction in the nifedipine $(20 \mu \mathrm{g} / \mathrm{ml})$ concentration was not significantly different from that in the diltiazem $\left(3 \times 10^{-7} \mathrm{~mol} / \mathrm{l}\right)$ concentration (Fig. 4).

\section{Discussion}

This study suggests that lipid emulsion attenuates the vasodilation evoked by a toxic dose of calcium channel blocker through the partitioning of the blocker into the lipid phase of the lipid emulsion. The major findings of this study are as follows: 1) The vasodilation produced by a mixture of lipid emulsion $(0.998 \%)$ and either bepridil or verapamil was not significantly different from the vasodilation produced by the corresponding CAE. 2) The relative inhibitory potency of lipid emulsion on the maximal vasodilation produced by each calcium channel blocker alone or calcium channel blocker concentration in decreasing order was as follows: bepridil > verapamil > nifedipine or diltiazem.

When a lipid emulsion containing a specific drug is ultracentrifuged, this lipid emulsion can be divided into three parts: the upper lipid phase, the middle interface composed of phospholipids, and the lower CAE (Sila-on et al. 2008). The magnitude of partitioning of a specific drug into the lipid phase of lipid emulsion is reportedly affected by the lipid solubility and distribution volume of the drug, as well as the temperature and pH (Mazoit et al. 2009; French et al. 2011b; Ruan et al. 2012). Ultracentrifugation of lipid emulsions containing drugs with variable lipid solubility results in greater absorption of highly lipid-soluble drugs in the upper lipid phase than of less lipid-soluble drugs and subsequently leads to a relatively low concentration of highly lipid-soluble drugs in the lower CAE, which seems to be equivalent to the low concentration of highly lipid-soluble drugs in the plasma (French et al. 2011b). The inhibitory effect of the mixture of lipid emulsion and either bepridil or verapamil on the maximal vasodilation induced by a toxic dose of either bepridil or verapamil alone was not significantly different from that of the

A

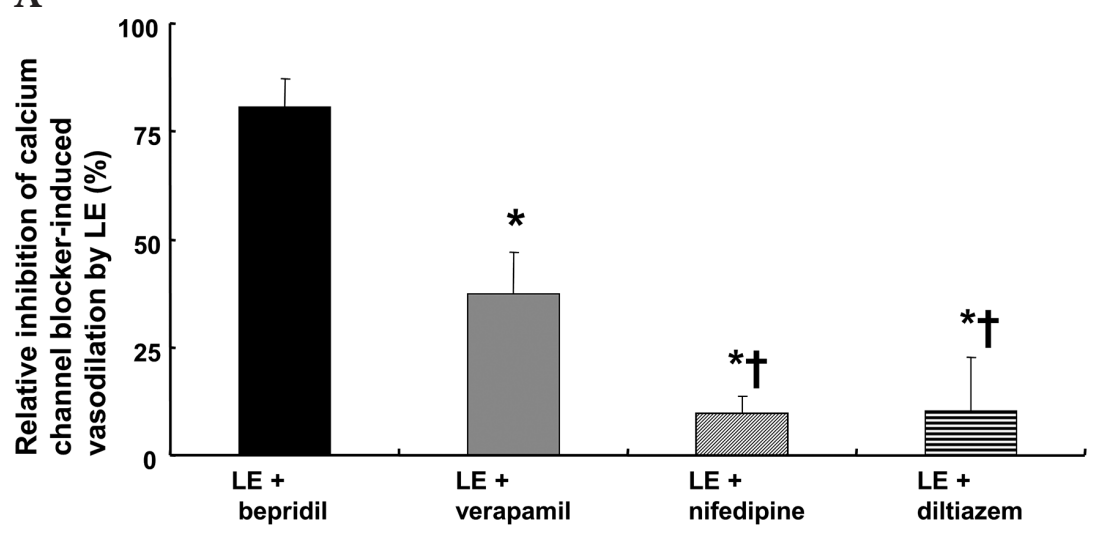

B

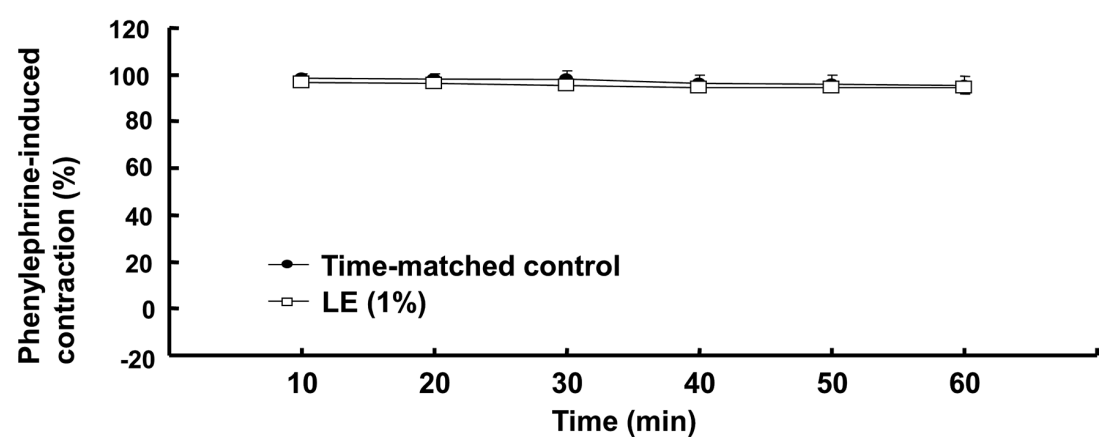

Figure 3. A. Relative inhibitory effect of the lipid emulsion (LE, $0.998 \%$ ) on the vasodilation induced by bepridil $\left(10^{-5} \mathrm{~mol} / \mathrm{l}\right.$, $n=7)$, verapamil $\left(10^{-7} \mathrm{~mol} / \mathrm{l}, n=7\right)$, nifedipine $\left(3 \times 10^{-7} \mathrm{~mol} / \mathrm{l}, n=7\right)$ and diltiazem $\left(3 \times 10^{-7} \mathrm{~mol} / \mathrm{l}, n=7\right)$ alone in isolated endothelium-denuded rat aortas precontracted with $10^{-7} \mathrm{~mol} / \mathrm{l}$ phenylephrine. The data are expressed as a percent difference in maximal vasodilation (evoked by the calcium channel blocker alone and a mixture of the LE and calcium channel blocker) divided by the maximal vasodilation evoked by calcium channel blocker alone. Data are shown as the mean $\pm \mathrm{SD}$; $n$ indicates the number of biological replicates (independent experiments). ${ }^{*} p<0.001$ versus LE $(0.998 \%)$ + bepridil $\left(10^{-5} \mathrm{~mol} / \mathrm{l}\right) ; \dagger p<0.001$ versus $\mathrm{LE}$ $(0.998 \%)+$ verapamil $\left(10^{-7} \mathrm{~mol} / \mathrm{l}\right)$. B. Effect of LE $(n=7)$ on the contraction induced by phenylephrine $\left(10^{-7} \mathrm{~mol} / \mathrm{l}\right)$ in isolated endothelium-denuded rat aorta. Data are expressed as the percentage of phenylephrineinduced contraction and shown as the mean $\pm \mathrm{SD} ; n$ indicates the number of biological replicates (independent experiments). 


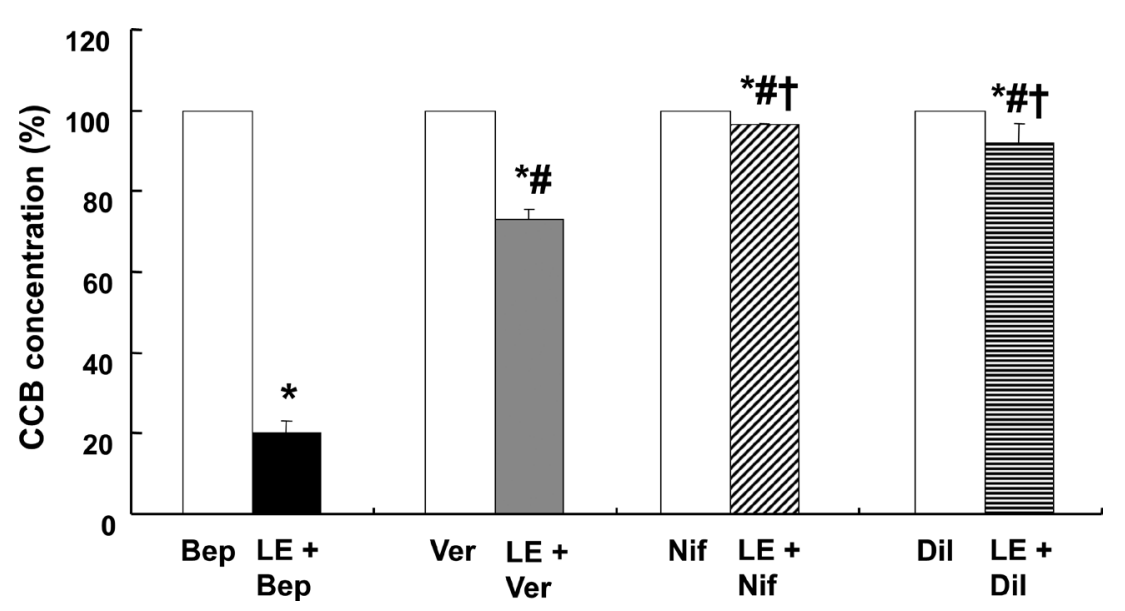

Figure 4. The effect of lipid emulsion (LE, $1 \%)$ on the concentration of calcium channel blocker (CCB; $10^{-5} \mathrm{~mol} / \mathrm{l}$ bepridil (Bep), $n=5 ; 10^{-7} \mathrm{~mol} / \mathrm{l}$ verapamil (Ver), $n=4$; $20 \mu \mathrm{g} / \mathrm{ml}$ nifedipine (Nif), $n=5$; and $3 \times$ $10^{-7} \mathrm{~mol} / \mathrm{l}$ diltiazem (Dil), $\left.n=4\right)$ in the Krebs solution. Data are shown as the mean $\pm \mathrm{SD}$ and expressed as the percentage of the concentration of each CCB alone (Bep, Ver, Nif and Dil); $n$ indicates the number of independent experiments. ${ }^{*} p<0.001$ versus each CCB alone (Bep, Ver, Nif and Dil); $\# p<0.001$ versus $\mathrm{LE}+\mathrm{Bep} ; \dagger p<0.001$ versus LE + Ver. corresponding CAE (Fig. 2A and B). In the case of nifedipine, the inhibitory effect of the lipid emulsion and nifedipine mixture on the maximal vasodilation induced by a toxic dose of nifedipine alone was less than that of the corresponding CAE (Fig. 2C). This greater inhibitory effect of the CAE may be ascribed to the chemical properties of nifedipine. As nifedipine is chemically unstable, an ultracentrifuge-induced physiochemical alteration of nifedipine may produce lower concentrations of nifedipine in the CAE (Kleinbloesem et al. 1984; Suzuki et al. 1985). However, the mixture of lipid emulsion and diltiazem or the corresponding CAE had no significant inhibitory effect on the maximal vasodilation induced by the toxic dose of diltiazem (Fig. 2D). Calcium channel blockers produce vasodilation mainly via the blockade of calcium influx via calcium channels, such as voltage-operated calcium channels in the plasma membrane (Akata 2007). The reported order of uptake of calcium channel blockers into rat aortic smooth muscle cells is as follows: bepridil > verapamil $>$ nifedipine or diltiazem (Pang and Sperelakis 1984). Therefore, as calcium channel blockers with high lipid solubility seem to be more absorbed into lipid emulsions than calcium channel blockers with low lipid solubility (Fig. 4), the mixture of the lipid emulsion with either bepridil or verapamil and the corresponding CAE may penetrate into the cell and cell membrane less than bepridil or verapamil alone, leading to less vasodilation via less calcium influx blockade. In an in vivo state, the calcium channel blocker absorbed into the lipid emulsion, which is a so-called lipid shuttle, may be delivered to the liver for detoxification (Weinberg 2012; Ok et al. 2018a). In the case of bepridil, which has the highest lipid solubility, the magnitude $(81 \pm 5 \%)$ of the lipid emulsion-mediated inhibition in vasodilation induced by bepridil seemed to be correlated with that of the lipid emulsion-mediated reduction in bepridil concentration $(80 \pm 3 \%)$. However, in the case of other calcium channel blockers, the lipid emulsion-mediated inhibition of vasodilation was not perfectly correlated with the reduction in the calcium channel blocker concentration. The magnitude of the lipid emulsion-mediated reduction in both the vasodilation induced by verapamil and the verapamil concentration was $37 \pm 10 \%$ and $27 \pm 2 \%$, respectively. In addition, the magnitude of the lipid emulsion-mediated reduction in both the vasodilation induced by nifedipine $\left(3 \times 10^{-7} \mathrm{~mol} / \mathrm{l}\right)$ and the nifedipine concentration $(20 \mu \mathrm{g} /$ $\mathrm{ml}$ ) was $10 \pm 4 \%$ and $3.5 \pm 0.3 \%$, respectively. Furthermore, the inhibitory effect of lipid emulsion on diltiazem-induced vasodilation was not significant $(10 \pm 13 \%)$, whereas lipid emulsion (1\%) decreased the diltiazem concentration by 8 $\pm 5 \%$. This difference between the lipid emulsion-mediated inhibition of vasodilation and the lipid emulsion-mediated reduction in the concentration of each calcium channel blocker may be due to the following putative causes: First, the potency of calcium channel blockers to produce vasodilation in isolated endothelium-denuded rat aortas precontracted with phenylephrine is as follows: nifedipine > verapamil $>$ diltiazem (Ok et al. 2018b). Second, lipid emulsion reduced the diltiazem concentration but potentially not to a level to increase calcium influx. Conversely, lipid emulsion only slightly reduced the nifedipine concentration, but the significant lipid emulsion-mediated inhibition of vasodilation induced by nifedipine may be due to both a greater potency of nifedipine to produce vasodilation compared with that of other calcium channel blockers and the different concentration $\left(20 \mu \mathrm{g} / \mathrm{ml}\right.$ versus $\left.3 \times 10^{-7} \mathrm{~mol} / \mathrm{l}\right)$ (Ok et al. 2018b). Third, the relationship between the amount of calcium channel blocker in the cells and the potency to inhibit calcium influx may not be a perfectly proportional linear relationship and may be dependent on the position of the calcium channel blocker dose-response curves. Thus, further study regarding the relationship between the lipid emulsion-induced reduction in calcium channel blocker concentration and the lipid emulsion-mediated attenuation of the vasodilation induced by the calcium channel blocker is needed. 
Similar to previous reports, lipid emulsion (1\%) reduced the verapamil concentration by $27 \pm 2 \%$ (French et al. 2011a; Kryshtal et al. 2016). In addition, the magnitude of the lipid emulsion-induced reduction in calcium channel blocker concentration was as follows: bepridil $(80 \pm 3 \%)>$ verapamil $(27 \pm 2 \%)>$ diltiazem $(8 \pm 5 \%)$ or nifedipine $(3.5 \pm 0.3)$. The reported oil/Ringer partition coefficient of calcium channel blockers $\left(10^{-6} \mathrm{M}\right)$, including bepridil, verapamil, nifedipine and diltiazem, was reported as follows: bepridil (32.9 \pm 3.1$)$, verapamil $(17.0 \pm 0.5)$, nifedipine $(2.30 \pm 0.03)$ and diltiazem $(0.02 \pm 0.00)$ (Pang and Sperelakis. 1984). Although lipid solubility is higher in nifedipine than in diltiazem, the difference in the lipid emulsion-mediated reduction in the nifedipine and diltiazem concentration was not significant in the present experiment. This lack of a significant difference may be associated with the approximately 190 times higher concentration of nifedipine $\left(20 \mu \mathrm{g} / \mathrm{ml}: 5.7 \times 10^{-5} \mathrm{~mol} / \mathrm{l}\right)$ than of diltiazem $\left(3 \times 10^{-7} \mathrm{~mol} / \mathrm{l}\right)$.

Taken together, these results suggest that vascular collapse (severe vasodilation) induced by a toxic dose of verapamil is relatively more responsive to lipid emulsion treatment than that induced by other clinically available calcium channel blockers. However, the extrapolation of this in vitro study on the clinical cardiovascular collapse induced by toxic doses of calcium channel blockers has several limitations. First, the calcium channel blocker concentration was only measured in the CAE in the current study. To prove the degree of partitioning of the calcium channel blocker into the upper lipid phase and lower CAE (upper lipid phase-induced entrapment of calcium channel blocker), the calcium channel blocker concentration should be simultaneously measured in both the upper lipid phase and lower CAE. Second, the ultracentrifugation-induced physicochemical alterations in the mixtures of lipid emulsion and each calcium channel blocker may affect the results of the current study. Third, most cases of intoxication caused by a toxic dose of calcium channel blocker are induced by oral administration, whereas the experimental conditions used in the present study are more similar to intravenous calcium channel blocker administration. Thus, the pharmacokinetics induced by oral administration of calcium channel blockers may modify the results of the current study.

In conclusion, lipid emulsion inhibits the maximal vasodilation induced by a toxic dose of calcium channel blockers by inducing partitioning of the blockers into the lipid phase of the lipid emulsion. In addition, the magnitude of partitioning of calcium channel blockers by lipid emulsions in decreasing order was as follows: bepridil > verapamil > nifedipine or diltiazem.

Acknowledgement. This research was supported by Basic Science Research Program through the National Research Foundation of Korea (NRF) funded by the Ministry of Science, ICT \& Future
Planning (2015R1C1A1A01054105). This research was supported by Basic Science Research Program through the National Research Foundation of Korea (NRF) funded by the Ministry of Education (NRF-2016R1D1A1B03930451).

Conflicts of interest. The authors declare that there is no conflict of interest.

\section{References}

Akata T (2007): General anesthetics and vascular smooth muscle: direct actions of general anesthetics on cellular mechanisms regulating vascular tone. Anesthesiology 106, 365-391 https://doi.org/10.1097/00000542-200702000-00026

Cao D, Heard K, Foran M, Koyfman A (2015): Intravenous lipid emulsion in the emergency department: a systematic review of recent literature. J. Emerg. Med. 48, 387-397 https://doi.org/10.1016/j.jemermed.2014.10.009

Charan J, Kantharia ND (2013): How to calculate sample size in animal studies? J. Pharmacol. Pharmacother. 4, 303-306 https://doi.org/10.4103/0976-500X.119726

Chung DM, Chung YC, Maeng PJ, Chun HK (2011): Regioselective deglycosylation of onion quercetin glucosides by Saccharomyces cerevisiae. Biotechnol. Lett. 33, 783-786 https://doi.org/10.1007/s10529-010-0501-8

Ding Y, Vaziri ND (2000): Nifedipine and diltiazem but not verapamil up-regulate endothelial nitric-oxide synthase expression. J. Pharmacol. Exp. Ther. 292, 606-609

Fettiplace MR, Akpa BS, Rubinstein I, Weinberg G (2015): Confusion about infusion: rational volume limits for intravenous lipid emulsion during treatment of oral overdoses. Ann. Emerg. Med. 66, 185-188 https://doi.org/10.1016/j.annemergmed.2015.01.020

French D, Armenian P, Ruan W, Wong A, Drasner K, Olson KR, Wu AH (2011a): Serum verapamil concentrations before and after Intralipid ${ }^{\oplus}$ therapy during treatment of an overdose. Clin. Toxicol. (Phila) 49, 340-344 https://doi.org/10.3109/15563650.2011.572556

French D, Smollin C, Ruan W, Wong A, Drasner K, Wu AH (2011b): Partition constant and volume of distribution as predictors of clinical efficacy of lipid rescue for toxicological emergencies. Clin. Toxicol. (Phila) 49, 801-809 https://doi.org/10.3109/15563650.2011.617308

Hori K, Matsuura T, Tsujikawa S, Mori T, Kuno M, Nishikawa $\mathrm{K}$ (2015): The significant contribution of the partitioning effect in lipid resuscitation for bupivacaine-induced cardiotoxicity: evaluation using centrifuged solution in vivo and in isolated hearts. Br. J. Anaesth. 115, 935-937 https://doi.org/10.1093/bja/aev386

Kleinbloesem CH, Van Harten J, Van Brummelen P, Breimer DD (1984) Liquid chromatographic determination of nifedipine in plasma and of its main metabolite in urine. J. Chromatogr. 308, 209-216 https://doi.org/10.1016/0378-4347(84)80210-8

Kryshtal DO, Dawling S, Seger D, Knollmann BC (2016): In vitro studies indicate intravenous lipid emulsion acts as lipid sink in verapamil poisoning. J. Med. Toxicol. 12, 165-171 
https://doi.org/10.1007/s13181-015-0511-y

Lee SH, Kang D, Ok SH, Kwon SC, Kim HJ, Kim EJ, Hong JM, Kim JY, Bae SI, An S, Sohn JT (2018): Linoleic acid attenuates the toxic dose of bupivacaine-mediated reduction of vasodilation evoked by the activation of adenosine triphosphate-sensitive potassium channels. Int. J. Mol. Sci. 19, e1876 https://doi.org/10.3390/ijms19010187

Liang CW, Diamond SJ, Hagg DS (2011): Lipid rescue of massive verapamil overdose: a case report. J. Med. Case Rep. 5, 399 https://doi.org/10.1186/1752-1947-5-399

Mazoit JX, Le Guen R, Beloeil H, Benhamou D (2009): Binding of long-lasting local anesthetics to lipid emulsions. Anesthesiology 110, 380-386 https://doi.org/10.1097/ALN.0b013e318194b252

Ok SH, Lee SH, Yu J, Park J, Shin IW, Lee Y, Cho H, Choi MJ, Baik J, Hong JM, et al. (2015): Lipid emulsion attenuates acetylcholine-induced relaxation in isolated rat aorta. Biomed. Res. Int. 2015, 871545 https://doi.org/10.1155/2015/871545

Ok SH, Hong JM, Lee SH, Sohn JT (2018a): Lipid emulsion for treating local anesthetic systemic toxicity. Int. J. Med. Sci. 15, 713-722

https://doi.org/10.7150/ijms.22643

Ok SH, Shin IW, Lee SH, Park J, Woo MS, Hong JM, Kim J, Sohn JT (2018b): Lipid emulsion alleviates the vasodilation and mean blood pressure decrease induced by a toxic dose of verapamil in isolated rat aortae and an in vivo rat model. Hum. Exp. Toxicol. 37, 636-646 https://doi.org/10.1177/0960327117721963

Ok SH, Sohn JT (2018); Amlodipine toxicity and lipid emulsion. Korean J. Anesthesiol. 71, 491-492 https://doi.org/10.4097/kja.d.18.00126

Pang DC, Sperelakis N (1984): Uptake of calcium antagonistic drugs into muscles as related to their lipid solubilities. Biochem. Pharmacol. 33, 821-826 https://doi.org/10.1016/0006-2952(84)90468-4

Presley JD, Chyka PA (2013): Intravenous lipid emulsion to reverse acute drug toxicity in pediatric patients. Ann. Pharmacother. 47, 735-743 https://doi.org/10.1345/aph.1R666
Ruan W, French D, Wong A, Drasner K, Wu AH (2012): A mixed (long- and medium-chain) triglyceride lipid emulsion extracts local anesthetic from human serum in vitro more effectively than a long-chain emulsion. Anesthesiology 116, 334-339 https://doi.org/10.1097/ALN.0b013e318242a5f1

Sampson CS, Bedy SM (2015): Lipid emulsion therapy given intraosseously in massive verapamil overdose. Am. J. Emerg. Med. 33, 1844.e1 https://doi.org/10.1016/j.ajem.2015.04.061

Schulz M, Schmoldt A (2003): Therapeutic and toxic blood concentrations of more than 800 drugs and other xenobiotics. Pharmazie 58, 447-474

Sila-on W, Vardhanabhuti N, Ongpipattanakul B, Kulvanich P (2008): Influence of incorporation methods on partitioning behavior of lipophilic drugs into various phases of a parenteral lipid emulsion. AAPS Pharm. Sci. Tech. 9, 684-692 https://doi.org/10.1208/s12249-008-9089-x

St-Onge M, Dubé PA, Gosselin S, Guimont C, Godwin J, Archambault PM, Chauny JM, Frenette AJ, Darveau M, Le Sage N, et al. (2014): Treatment for calcium channel blocker poisoning: a systematic review. Clin. Toxicol. (Phila) 52, 926-944 https://doi.org/10.3109/15563650.2014.965827

Suzuki H, Fujiwara S, Kondo S, Sugimoto I (1985): Determination of nifedipine in human plasma by high-performance liquid chromatography with electrochemical detection. J. Chromatogr. 341, 341-347 https://doi.org/10.1016/S0378-4347(00)84047-5

Wagner M, Zausig YA, Ruf S, Rudakova E, Gruber M, Graf BM, Volk T (2014): Lipid rescue reverses the bupivacaine-induced block of the fast $\mathrm{Na}+$ current (INa) in cardiomyocytes of the rat left ventricle. Anesthesiology 120, 724-736 https://doi.org/10.1097/ALN.0b013e3182a66d4d

Weinberg GL (2012): Lipid emulsion infusion: resuscitation for local anesthetic and other drug overdose. Anesthesiology 117, 180-187 https://doi.org/10.1097/ALN.0b013e31825ad8de

Received: October 22, 2018

Final version accepted: February 19, 2019 